\title{
Determinantes de la estructura de capital en la pequeña y mediana empresa familiar en México $^{1}$
}

José García Berumen

González

Consultor independiente de empresas

armandogberumen@gmail.com

\section{Pablo García Soto}

Consultor independiente de empresas

pgs10@hotmail.com

\section{Rogerio Domenge}

Muñoz

Instituto Tecnológico Autóno-

mo de México

domenge@itam.mx

\section{Resumen}

Este trabajo examina los determinantes internos que influyen en la estructura de capital de las pequeñas y medianas empresas familiares en México (Pymef). Se sugiere que el tamaño y la antigüedad de la empresa, la formalidad en la planeación administrativa y estratégica, la actitud hacia el control familiar y la edad del director influyen en las decisiones del tipo de fuentes de financiamiento por utilizar. A través de un análisis de trayectorias se validan estadísticamente varias hipótesis tomando como base una muestra de 240 Pymef mexicanas. Los resultados indican relaciones significativas entre tamaño y deuda, así como entre edad del director, capital social y utilidades acumuladas. Asimismo, aplicando el análisis por sector de actividad y por tamaño, se encontró evidencia empírica sobre las influencias entre el tamaño de la empresa y el capital social, así como la formalidad de la planeación administrativa y estratégica con la deuda. Los resultados sugieren líneas de investigación de las Pymef mexicanas.

Palabras clave: estructura de capital, pequeña y mediana empresa familiar (Pymef), ecuaciones estructurales, México. 


\title{
Capital structure determinants in small and medium size Mexican family firms
}

\begin{abstract}
This paper examines the determinants of capital structure in family-owned small and medium-sized enterprises in Mexico. We suggest that the size, age, managerial planning, family control and the age of their CEO or owner influence financing decisions. The study's hypotheses are tested by analyzing a survey data collected from 240 Mexican SMEs with Path Analysis. We found that the size of the firm has a positive relationship with debt; the age of SME's owner has an influence on the equity and retained earnings funding. Through splitting the data by sectors and size, we also found two additional relationships: between size and equity, and between managerial planning formality and debt. Our findings suggest directions for further research on Mexican family-owned SMEs.
\end{abstract}

Keywords: capital structure, small and medium-sized enterprises, structural equation model, Mexico.

En México, el 99\% del total de empresas, que equivalen a unos cuatro millones, son catalogadas como micro, pequeñas y medianas empresas (OCDE, 2007; INEGI, 2006). Asimismo, en el año 2006, estas empresas generaron más de la mitad del Producto Interno Bruto (PIB) nacional y emplearon casi tres cuartas partes de la población económicamente activa. De igual manera, la importancia de las empresas familiares en México es evidente al observar que aproximadamente nueve de cada diez compañías son de carácter familiar (Kuhlman, 1997; Belausteguigoitia, 2002).

La estructura financiera empresarial ha sido objeto de estudio de varias teorías que proponen básicamente la existencia de una estructura de capital óptima que maximiza el valor de una compañía. Sin embargo, la mayoría de los estudios evalúan empresas grandes, asumiendo mercados perfectos y un comportamiento económico racional (Modigliani y Miller, 1958), lo que limita y cuestiona su aplicación en las Pyme. De igual forma, existen muchos factores que determinan la viabilidad de una empresa y uno de ellos es el tipo de estructura de capital que se tenga (Jones, 1979; Smith, 1986), la cual es resultado tanto de las decisiones financieras en la propia empresa - llevadas a cabo día con día- como de las circunstancias externas que la rodean —como la disponibilidad y características del crédito y del

${ }^{1}$ Se agradece a la Asociación Mexicana de Cultura, A.C. por el apoyo en la elaboración de la presente investigación. 
capital. Esta decisión tiene repercusiones en el crecimiento de la empresa, en los flujos de efectivo y, en general, en la rentabilidad de la misma.

Debido a la constante renuencia de los empresarios familiares por compartir información (Brockhaus, 1994) —entre otras razones- no han sido estudiados con profundidad los determinantes clave de la estructura de capital de las Pymef en general, y más específicamente en México. En consecuencia, los investigadores no pueden explicar satisfactoriamente cómo es que los administradores eligen entre las distintas estructuras de capital posibles, lo cual es una de tantas limitantes para que el sector financiero elabore productos financieros que se adecuen correctamente a las distintas necesidades y particularidades de las Pymef mexicanas (Domenge, 2008; García y García, 2007). Esta investigación tiene como objetivo identificar los factores internos que determinan las decisiones respecto a la estructura de capital de este tipo de empresas.

\section{Antecedentes teóricos}

El tema de estructura de capital ha sido estudiado por varios autores y desde distintas perspectivas. Debido a la gran cantidad de teorías desarrolladas, es conveniente organizarlas en grupos. En este trabajo se hará uso de la siguiente clasificación: teorías de la irrelevancia, teorías del equilibrio, modelos estáticos y dinámicos, y teorías de asimetría de la información.

A partir de 1958, con el trabajo de Merton H. Miller y Franco Modigliani, se han ido desarrollando varias teorías sobre la estructura de capital. Ellos propusieron que la decisión entre el financiamiento mediante deuda y capital no tenía efectos significativos en el valor de la empresa siempre y cuando se cumpliera con ciertos supuestos: un mercado perfecto de capitales, el costo de la deuda es constante en el tiempo, se reparten el $100 \%$ de las utilidades y las expectativas del mercado son homogéneas. Sin embargo, la restricción más importante que se encontró en esta teoría es su limitada aplicación en las compañías pequeñas y empresas familiares (Chaganti et al., 1995).

Por otro lado, las teorías del equilibrio proponen que la estructura de capital se puede determinar por medio de los intercambios que existen entre las ventajas fiscales asociadas al financiamiento a través de la deuda y las desventajas del endeudamiento excesivo, como los costos de quiebra y de insolvencia financiera (Prasad 
et al., 2001). Existen dos ramas de dicha teoría: tradicional y moderna (modelos estáticos y dinámicos).

La primera versión de la teoría del equilibrio supone que el costo del financiamiento con deuda es menor al del capital; por lo tanto, se recomendaba a las empresas aumentar su posición de deuda para que el costo de capital se viera disminuido. Sin embargo, este proceso no era sostenible porque al aumentar excesivamente la proporción de deuda la posibilidad de incurrir en insolvencia financiera también se incrementa. Una de las limitantes de la teoría tradicional es que no considera en su totalidad algunas de las imperfecciones del mercado como los impuestos, los costos de agencia y los costos relacionados con la quiebra.

Por otro lado, las versiones modernas de la teoría del equilibrio incorporan en los modelos las limitantes antes mencionadas, sosteniendo que existe un equilibrio óptimo donde el costo del capital es mínimo y el valor de la firma se maximiza. Los modelos estáticos tienen como objetivo principal proveer información sobre cómo determinan las empresas su estructura de capital. Sin embargo, son limitativos porque se concentran en sólo uno o dos elementos del problema e ignoran otros (Titman y Tsyplakov, 2005); otra limitante es su enfoque en un solo periodo, que ignora las decisiones de reestructuración como respuesta a cambios en el tiempo del valor de los activos (Fischer et al., 1989, citado en Sander, 1998). Por su parte, los modelos dinámicos surgen como respuesta a la búsqueda de un modelo que muestre la evolución de los cambios en la estructura de capital a través del tiempo (Titman y Tsyplakov, 2005). En general, estos modelos hacen uso de la intuición utilizada en la teoría de opciones reales, que sugiere que es óptimo aplazar cualquier decisión costosa en un entorno bajo incertidumbre; por lo tanto, las empresas no toman decisiones de financiamiento de manera inmediata, lo que significa que aun cuando cuenten con una estructura de capital objetivo se acercan a ella lentamente.

Por último, las teorías de asimetría de información sugieren que las empresas tienen un orden de preferencia particular en sus opciones de financiamiento (Myers, 1984), por lo que el seguir cierta jerarquía en dichas opciones maximizará el valor de la empresa, lo que contrasta con la teoría del equilibrio que propone una proporción objetivo de deuda-capital. De acuerdo con Myers (1984), las empresas generalmente prefieren el financiamiento mediante capital interno a deuda, la deuda a la deuda convertible y la deuda convertible al capital externo. En particular, debido 
a la existencia de información asimétrica entre la compañía y los inversionistas (acreedores), el costo relativo del financiamiento dependerá de la opción elegida.

Para ilustrar la lógica de la jerarquía antes mencionada, se observa que cuando se utiliza el financiamiento interno mediante las utilidades acumuladas o las nuevas contribuciones de los accionistas o dueños existentes, éstos cuentan con mayor información que los nuevos, si es que los hubiera. Por lo tanto, los últimos demandarían un mayor retorno sobre la inversión; esto trae como resultado que el incremento de capital a través de nuevos inversionistas sea más cara que el financiamiento interno. Asimismo, un argumento similar puede ser utilizado para explicar la preferencia por el financiamiento interno sobre la deuda externa.

Así, se han desarrollado varios modelos que proponen diversos determinantes (externos e internos) de la estructura de capital. Sin embargo, la mayoría de ellos han sido estudiados basándose en datos de empresas públicas y en países desarrollados. En cambio, solamente muy pocos han considerado los atributos de las pequeñas y medianas empresas en países en desarrollo para buscar los factores que determinen su estructura financiera. Por ejemplo, el modelo desarrollado por Titman y Wessels (1988) ha sido utilizado para aproximar los determinantes de la estructura de capital por medio de un análisis de las variables cuantitativas internas. Ellos identificaron como determinantes de dicha estructura a la particularidad del producto, la industria y el tamaño de la compañía. Aunque el estudio de dichos autores fue novedoso, también puede parecer algo limitado, ya que sólo se estudiaron empresas públicas en Estados Unidos.

Existen investigaciones empíricas recientes que, además de analizar los determinantes de la estructura con atributos internos de las empresas (tamaño de la compañía, crecimiento y rendimiento esperado, valor de activos, industria, riesgos del negocio, depreciación y estructura de control), estudian el efecto de los factores externos (reglamentos fiscales, regulaciones del mercado de capital, inflación, déficit presupuestario del gobierno, ciclo del negocio y de la industria, y ciclo del mercado de valores). Un ejemplo fue el estudio comparativo efectuado en los países del grupo G-7 (considerados, en ese entonces, los siete países más industrializados del mundo: Alemania, Canadá, Estados Unidos, Francia, Gran Bretaña, Italia y Japón) por Rajan y Zingales (1995) con el objetivo de conocer la importancia de algunos factores como el tamaño y la rentabilidad en el tipo de fuente de financiamiento de la compañía. Ellos concluyeron que sí existe una similitud significativa entre los factores que determinan la estructura de capital en todos los países. 
Similarmente, Booth et al. (2001) llevaron a cabo una investigación comparativa en diez países con economías emergentes para demostrar que los factores determinantes de la estructura de capital no son particulares de las naciones desarrolladas y pueden ser aplicados de la misma manera. No obstante, también identificaron que las características de cada país generan diferencias significativas; por ejemplo, la percepción de las condiciones del entorno macroeconómico influye sobre las decisiones de apalancamiento, pues dependiendo del nivel riesgo-país, tanto las instituciones financieras como las compañías, tendrán cierto cuidado con el nivel de endeudamiento.

Aplicando las teorías anteriores en las Pyme, Chaganti et al. (1995) —en Estados Unidos - y Cassar y Holmes (2003) —en Australia — encontraron diferencias sustantivas entre la pequeña y la grande empresa. Jones (1979) identificó que la composición del capital inicial juega un papel importante en el éxito de la pequeña empresa; demasiada deuda al principio tiende a generar problemas de liquidez. Asimismo, Levin y Travis (1987) sugieren que en las pequeñas empresas las preferencias del administrador, con respecto a la elección entre deuda y capital, juegan un papel más importante que en las grandes organizaciones. Por su parte, Barton y Gordon (1987) sostienen que los altos directivos prefieren financiar las actividades de la pequeña empresa utilizando recursos generados por la misma. Existe evidencia empírica que indica que, en general, los dueños de los pequeños negocios desean mantener el control sobre las decisiones estratégicas (Shrivastava y Grant, 1985).

Chaganti et al. (1995) encontraron que la búsqueda del dueño por tener un empleo estable y su deseo por crear una empresa exitosa son los determinantes más importantes en la decisión de la fuente de capital. Estos resultados sugieren que las características personales del emprendedor juegan un papel clave en las decisiones de estructura de capital.

Cassar y Holmes (2003) utilizan la información previamente generada por estudios de carácter descriptivo para construir una investigación empírica con gran rigor científico. Los resultados muestran que la estructura de los activos, la rentabilidad y el crecimiento son determinantes importantes en el financiamiento y en la estructura de capital de las pequeñas y medianas empresas. Finalmente, demuestran que el análisis de la información indica que las teorías financieras tradicionales, típicamente desarrolladas para grandes empresas públicas, también pueden ser aplicadas a las Pyme australianas. 


\section{El modelo estructural}

La teoría indica que existe un complejo número de factores que influyen en las decisiones tomadas por los dueños de las Pymef para elegir la estructura de capital. Entre éstos destacan las características emprendedoras de los administradores, las experiencias pasadas del dueño con las estructuras de capital, los objetivos del negocio, el grado de control ejercido, las proporciones elegidas entre deuda y capital, el tamaño y antigüedad de la empresa, la aversión al riesgo del administrador. Sin embargo, dichos factores no se han hecho explícitos debido a que en la mayoría de los estudios realizados, ya sea en las Pyme que cotizan en el mercado accionario o no, dejan a un lado consideraciones y particularidades de la empresa familiar (Romano et al., 2000).

El modelo desarrollado por Romano et al. (2000) responde al vacío que existía, en el nivel mundial, en la literatura acerca de las fuentes de capital en las Pymef. En particular, el interés de los autores radica en identificar los factores que influyen en la elección de la estructura de capital por parte de los dueños-administradores de este tipo de empresas. Aunque el modelo propuesto en esta investigación tiene como referencia el de Romano et al. (2000), éste ha sido modificado, por un lado, para simplificar la recolección de datos debido a las dificultades en la obtención de información de las empresas familiares mexicanas y, por otro, para incluir hallazgos adicionales provenientes de la revisión de la literatura.

En la figura 1 se presenta el modelo propuesto para la presente investigación, donde se muestran los constructos por estudiar y las relaciones causales que se buscan corroborar.

\section{Variables dependientes}

Siguiendo la división propuesta en el modelo de Romano et al. (2000), el concepto de estructura de capital será representado por cuatro elementos: deuda, préstamos familiares, capital social y utilidades acumuladas, considerando - como Poutziouris et al. (1998) - que los dueños de las pequeñas empresas son adversos a fuentes de capital que vayan más allá de las antes mencionadas. 


\section{Figura 1}

Modelo causal de los determinantes de la estructura de capital para las empresas familiares en México

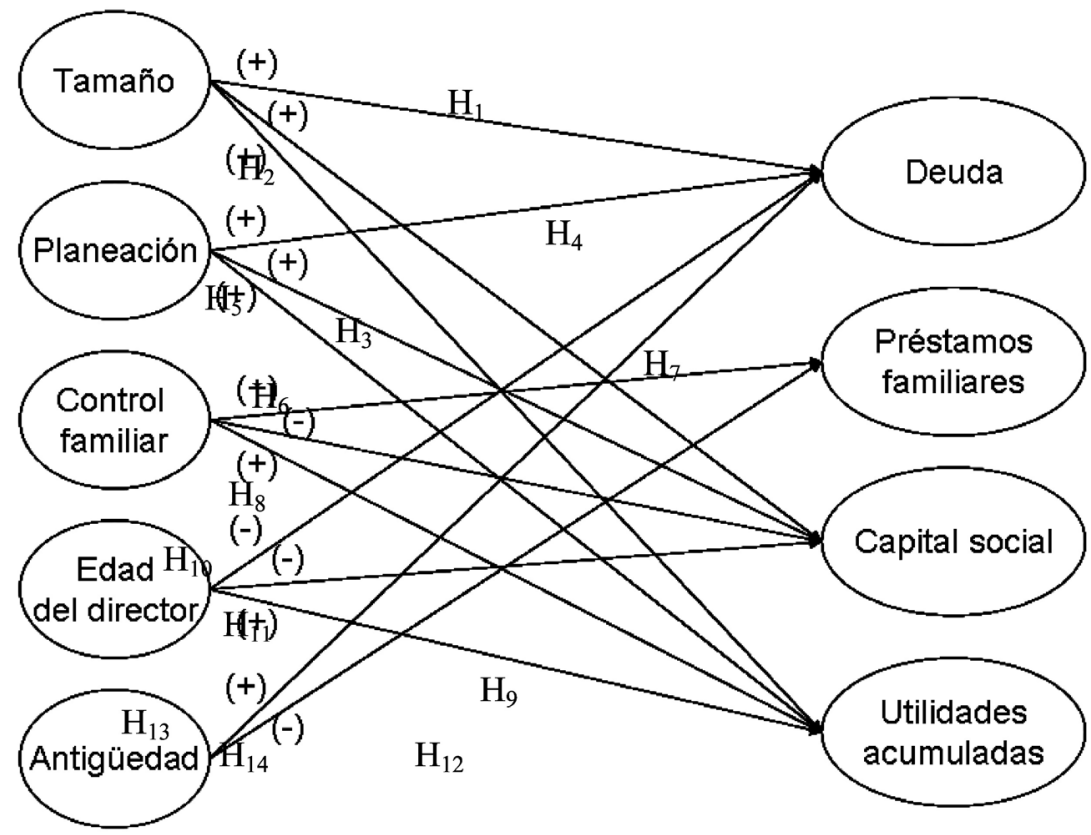

Deuda y préstamos familiares engloban todo tipo de obligaciones futuras a terceros contenidas en el pasivo, mientras que capital social y utilidades acumuladas enmarcan los rubros incluidos en el capital contable de una empresa. Para fines de la presente investigación, se reconoce como capital social las aportaciones efectuadas por los socios, en tanto que las utilidades acumuladas se refieren al capital generado por la empresa.

\section{Variables independientes}

Para definir las siguientes variables independientes y su inclusión dentro del modelo se partirá de la justificación teórica descrita por Romano et al. (2000), complementadas con las siguientes variables que fueron modificadas para la presente investigación.

- Tamaño de la empresa: A pesar de que Modigliani y Miller (1958) sostienen que el tamaño de la empresa no afecta significativamente la estructura de 
capital de la misma, existen varios estudios que demuestran un vínculo entre el tamaño y la estructura financiera (Berger y Udell, 1998; Hutchinson, 1995). Por ejemplo, Chittenden et al. (1996) encontraron que la teoría de asimetría de la información es particularmente relevante en las pequeñas empresas porque los costos asociados con el financiamiento externo son mayores para las pequeñas que para las grandes compañías. Por otro lado, Sonnenfeld y Spence (1989) sostienen que los negocios familiares tienen una razón deuda-capital baja para evitar dañar la reputación familiar, las garantías personales y la pérdida de su patrimonio en caso de insolvencia económica.

- Planeación administrativa y estratégica formal: Esta variable surgió de la combinación de tres conceptos utilizados por Romano et al. (2000): plan de negocios, objetivos del negocio y planes de crecimiento. La inclusión de esta variable en el modelo se justifica con las investigaciones de Chaganti et al., (1995), las cuales sostienen que la estrategia de una empresa puede ser una variable útil para explicar el comportamiento de la estructura de capital. Asimismo, es común que los dueños de las Pyme utilicen un plan de negocios, como documento de apoyo, para buscar fuentes de financiamiento externo como préstamos bancarios (Berger y Udell, 1998). En línea con esta propuesta, Harvey y Evans (1995) encontraron que los bancos enfatizan la importancia de un plan de negocios completo, relacionado positivamente con los préstamos obtenidos.

Para los fines de este trabajo, se considera que la planeación administrativa y estratégica formal consta de cinco elementos: misión y visión, plan estratégico, organigrama, manuales de descripción de puestos y capacitación. Se asume que un plan estratégico consiste en el desarrollo de una visión y misión, el establecimiento de objetivos y la formulación de una estrategia que, en conjunto, determinen la dirección de la empresa (Thompson y Strickland, 1999). Asimismo, de acuerdo con Smyrnios et al. (1998), la descripción del organigrama, los manuales de puestos y el entrenamiento de los administradores juegan un papel importante tanto en los objetivos del negocio como en el sistema de administración de una empresa familiar.

- Control familiar: Se refiere a la disposición de la familia a compartir el control de la empresa con externos. Se busca analizar, por un lado, la disposición de aceptar la intervención de externos a la familia en la empresa; 
por otro, evaluar los planes futuros sobre el control de la empresa (Smyrnios et al., 1998). Berger y Udell (1998) sostienen que los hechos relacionados con el control y la aversión al riesgo influyen en la elección de las fuentes de capital. Por su parte, Hutchinson (1995) sugiere que los emprendedores que tienen una alta preferencia por mantener la independencia del negocio utilizan capital social (mientras no involucre la participación de externos) o utilidades acumuladas como fuentes de financiamiento. En tanto que Storey (1994) indica que los dueños de las pequeñas empresas tienden a utilizar deuda debido a que se oponen a compartir el control del negocio.

- Edad del director general: Van der Wijst (1989) encontró que los emprendedores de mayor edad utilizan menos deuda y son más reacios que los jóvenes para aceptar participación de externos. Zimmerer y Scarborough (2002) sostienen que los años de experiencia profesional muchas veces definen si el director tiene el poder de visualizar, administrar e integrar las operaciones en una sinergia exitosa. Bertrand y Schoar (2002) muestran que los antecedentes y la preparación de los altos directivos pueden predecir sus decisiones financieras.

- Antigüedad de la empresa: De acuerdo con Dollinger (1995), las fuentes de capital dependen, en cierto grado, de la etapa en la que la empresa se encuentre, es decir, nacimiento, desarrollo o madurez. Las empresas en consolidación tienden a financiarse con capital social por las dificultades de acceder a préstamos bancarios, mientras que los negocios consolidados son capaces de apalancar sus activos con deuda. Por lo mismo, no es poco común para los emprendedores financiarse, primeramente, por medio de ahorros personales, amigos y parientes (Hutchinson, 1995; Delgado et al., 2006). Sin embargo, es importante señalar que existen investigaciones que indican que la tasa de crecimiento de las empresas también es un factor clave en la elección de financiamiento. Berger y Udell (1998) encontraron que las pequeñas compañías con altas tasas de crecimiento, las cuales operan en sectores altamente riesgosos, utilizan capital social para financiarse; mientras que las que crecen a un ritmo menor, deuda. Asimismo, concluyen que mientras la empresa madura, ésta comienza a confiar en otros métodos de financiamiento; primero en préstamos bancarios a corto plazo y después a largo plazo, hasta encontrarse lista para obtener capital externo e incluso volverse pública. 


\section{Con base en estos planteamientos y considerando el modelo de la figura 1, el cua- dro 1 resume las 14 hipótesis consideradas en esta investigación.}

\section{Cuadro 1 Hipótesis de trabajo}

\begin{tabular}{|c|c|}
\hline $\mathbf{H}_{1}$ & $\begin{array}{l}\text { El tamaño de la empresa familiar impacta positivamente } \\
\text { la deuda como fuente de financiamiento. }\end{array}$ \\
\hline $\mathbf{H}_{2}$ & $\begin{array}{l}\text { El tamaño de la empresa familiar impacta positivamente } \\
\text { el capital social como fuente de financiamiento. }\end{array}$ \\
\hline $\mathbf{H}_{3}$ & $\begin{array}{l}\text { El tamaño de la empresa familiar impacta positivamente } \\
\text { las utilidades acumuladas como fuente de financiamiento. }\end{array}$ \\
\hline $\mathbf{H}_{4}$ & $\begin{array}{l}\text { La planeación administrativa y estratégica formal de las empresas familiares } \\
\text { impacta positivamente la deuda como fuente de financiamiento. }\end{array}$ \\
\hline $\mathbf{H}_{5}$ & $\begin{array}{l}\text { La planeación administrativa y estratégica formal de las empresas familiares } \\
\text { impacta positivamente el capital social como fuente de financiamiento. }\end{array}$ \\
\hline $\mathbf{H}_{6}$ & $\begin{array}{l}\text { La planeación administrativa y estratégica formal de las empresas familiares } \\
\text { impacta positivamente las utilidades acumuladas como fuente de financiamiento. }\end{array}$ \\
\hline $\mathbf{H}_{7}$ & $\begin{array}{l}\text { El control familiar, ejercido por los dueños, impacta positivamente } \\
\text { los préstamos familiares como fuente de financiamiento. }\end{array}$ \\
\hline $\mathbf{H}_{8}$ & $\begin{array}{l}\text { El control familiar, ejercido por los dueños, impacta negativamente } \\
\text { el capital social como fuente de financiamiento. }\end{array}$ \\
\hline $\mathbf{H}_{9}$ & $\begin{array}{l}\text { El control familiar, ejercido por los dueños, impacta positivamente } \\
\text { las utilidades acumuladas como fuente de financiamiento. }\end{array}$ \\
\hline$H_{10}$ & $\begin{array}{l}\text { La edad del director impacta negativamente la deuda } \\
\text { como fuente de financiamiento. }\end{array}$ \\
\hline $\mathbf{H}_{11}$ & $\begin{array}{l}\text { La edad del director impacta negativamente el capital social } \\
\text { como fuente de financiamiento. }\end{array}$ \\
\hline$H_{12}$ & $\begin{array}{l}\text { La edad del director impacta positivamente las utilidades acumuladas } \\
\text { como fuente de financiamiento. }\end{array}$ \\
\hline $\mathbf{H}_{13}$ & $\begin{array}{l}\text { La antigüedad de la empresa familiar impacta positivamente la deuda } \\
\text { como fuente de financiamiento. }\end{array}$ \\
\hline$H_{14}$ & $\begin{array}{l}\text { La antigüedad de la empresa familiar impacta negativamente } \\
\text { los préstamos familiares como fuente de financiamiento. }\end{array}$ \\
\hline
\end{tabular}




\section{Método}

\section{Participantes}

La población de interés en esta investigación fueron las pequeñas y medianas empresas familiares mexicanas, utilizando los criterios oficiales de estratificación publicados por el Instituto Nacional de Estadística, Geografía e Informática (INEGI, 2006). Con el objetivo de aplicar el cuestionario, se buscó la cooperación de dos organismos que tuvieran relaciones directas con las Pymef: la Cámara de Comercio del Distrito Federal y Profit Consulting, S.C., en el Foro Nacional de Empresas Familiares.

La recolección de información se obtuvo mediante la aplicación de un total de 707 cuestionarios, de los cuales 240 fueron válidos (34\%) después de una depuración exhaustiva. Este número se encuentra dentro de lo recomendable (Hair et al., 1999) de por lo menos 200 sujetos, considerando las características del modelo propuesto en esta investigación, o como menciona Tenenhaus (2007) de por lo menos 100 sujetos, dependiendo también de la complejidad del modelo y del algoritmo por utilizar para estimar sus parámetros.

\section{Medición}

El diseño del cuestionario (Anexo 1) se basó en el modelo de la figura 2. Se diseñaron varios reactivos que pudieran medir cada uno de los constructos, los que se discutieron con académicos y se seleccionaron los más adecuados. Se llevó a cabo una prueba piloto del cuestionario entre empresarios familiares y finalmente se revisó y pulió. La figura 2 presenta el modelo por utilizar, que también incluye el de medición con sus indicadores o variables observadas y las variables latentes y sus relaciones estructurales de acuerdo con las hipótesis consideradas.

- Estructura de capital: se midió la proporción que cada una de las variables dependientes (deuda, préstamos familiares, capital social y utilidades acumuladas) representan del total (100\%) de las fuentes de financiamiento de la empresa. En cada una de las variables se utiliza una escala continua (Romano et al., 2000).

- Tamaño: generalmente se utilizan los activos netos totales y el valor estimado del negocio; sin embargo, dentro de los estudios de empresas familiares, 
muchas veces existen dificultades para obtener la cooperación de miembros de la familia para recabar información (Brockhaus, 1994). Debido a ello se buscó simplificar las preguntas, utilizando exclusivamente aquellas que pudieran ser contestadas con mayor facilidad. Finalmente, para estimar el tamaño se utilizó una medida compuesta por tres variables continuas: número de empleados (Scott, 1981), logaritmo natural de las ventas brutas anuales (Titman y Wessels, 1988) y número de establecimientos (Romano et al., 2000).

- Planeación administrativa y estratégica formal: se utilizó una medida de percepción compuesta por cinco variables observadas, medida cada una de éstas utilizando una escala Lickert con cinco puntos (Smyrnios et al., 1998). Las variables representan la percepción sobre la formalidad en la visión y misión, plan estratégico (Romano et al., 2000), organigrama, descripción de puestos y capacitación.

- Control familiar: se utilizaron cuatro variables observadas sobre la actitud hacia el control familiar, que se midieron por medio de una escala Lickert con cinco puntos (Smyrnios et al., 1998). Estas variables miden la actitud hacia la aceptación de accionistas no familiares, administrativos no familiares, contratar a empleados no familiares y la sucesión familiar.

- Edad del director: se utilizó una sola medida directa: la edad del director (Romano et al., 2000).

- Antigüedad: se utilizó una sola variable directa que mide el número de años que tiene la organización en el negocio (Romano et al., 2000). 
Figura 2

Modelo estructural

Relaciones entre variables observadas y latentes

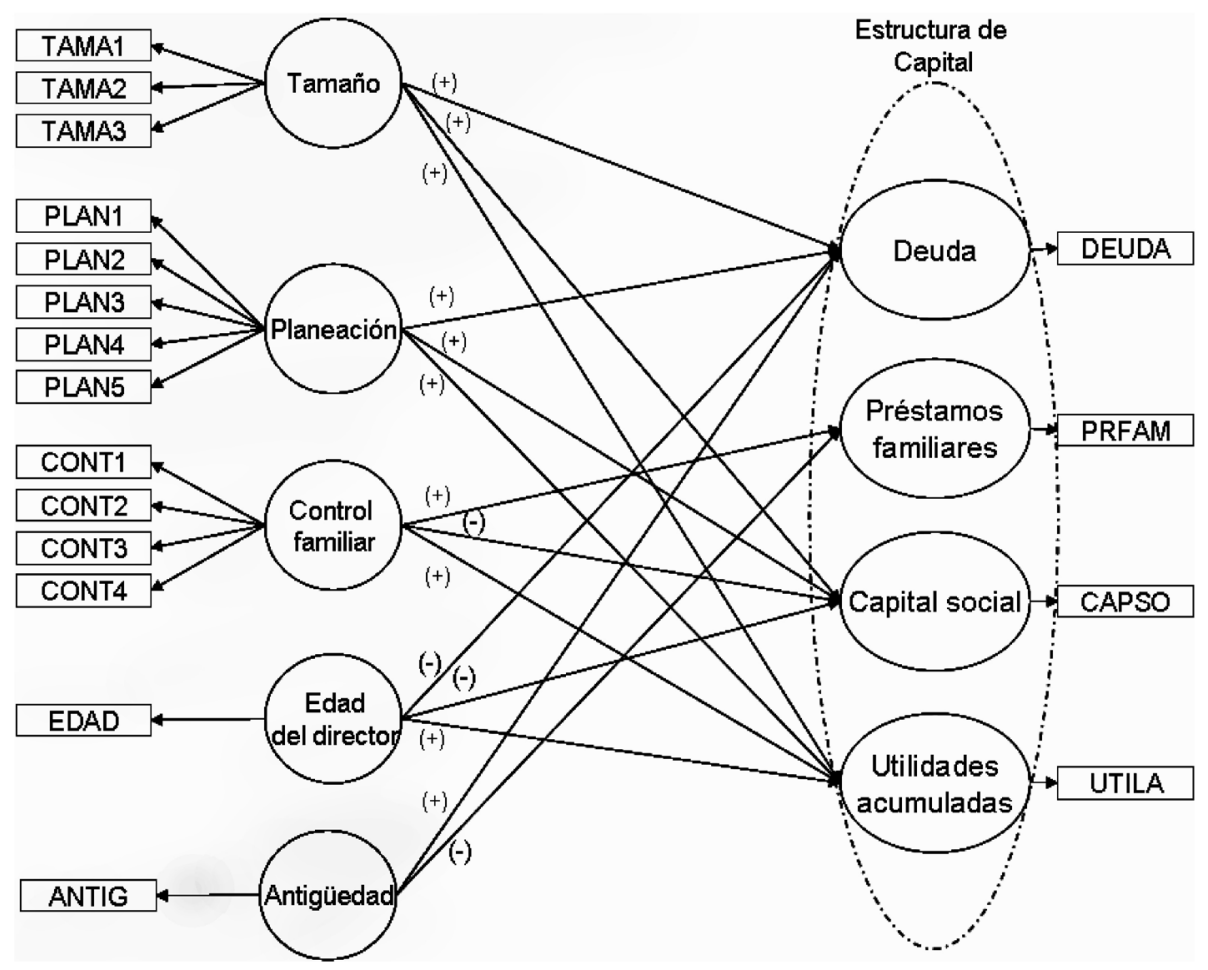

Los estadísticos descriptivos de la muestra se presentan en el cuadro 2, donde se observa la distribución de frecuencias para las variables puesto del encuestado, edad del director o gerente general, escolaridad del director o gerente general, el sector al cual pertenece la Pymef, las ventas anuales, las medias de los porcentajes de la estructura de capital real y la esperada o deseada.

Los directores o gerentes generales cuentan con una experiencia profesional media de 20 años; en tanto, las empresas tienen una media de antigüedad de existencia de 22 años y cuentan con un promedio de 43 empleados. 


\section{Cuadro 2}

\section{Estadísticos descriptivos de la muestra}

\begin{tabular}{|c|c|c|}
\hline \multirow{3}{*}{ Puesto del encuestado } & Dueños & $62 \%$ \\
\hline & Directores & $17 \%$ \\
\hline & Gerentes & $21 \%$ \\
\hline \multirow{5}{*}{ Edad del director/gerente } & 20-30 años & $6 \%$ \\
\hline & 31-40 años & $21 \%$ \\
\hline & 41-50 años & $29 \%$ \\
\hline & 51-60 años & $29 \%$ \\
\hline & Más de 60 años & $15 \%$ \\
\hline \multirow{5}{*}{$\begin{array}{l}\text { Escolaridad del } \\
\text { director/gerente }\end{array}$} & Secundaria & $5 \%$ \\
\hline & Preparatoria & $6 \%$ \\
\hline & Carrera técnica & $6 \%$ \\
\hline & Licenciatura & $48 \%$ \\
\hline & Posgrado & $26 \%$ \\
\hline \multirow{3}{*}{ Sector de la Pyme } & Comercio & $29.6 \%$ \\
\hline & Industria & $25.4 \%$ \\
\hline & Servicios & $45.0 \%$ \\
\hline \multirow{5}{*}{$\begin{array}{l}\text { Ventas anuales } \\
\text { (miles pesos) }\end{array}$} & Menos de 500 & $40 \%$ \\
\hline & $500-1,000$ & $18 \%$ \\
\hline & $1,000-15,000$ & $10 \%$ \\
\hline & $15,000-20,000$ & $5 \%$ \\
\hline & Más de 20,000 & $27 \%$ \\
\hline \multirow{4}{*}{$\begin{array}{l}\text { Estructura } \\
\text { de capital real }\end{array}$} & Capital social & $57 \%$ \\
\hline & Deuda (bancos, proveedores) & $17 \%$ \\
\hline & Utilidad & $19 \%$ \\
\hline & Préstamos familiares & $7 \%$ \\
\hline \multirow{6}{*}{$\begin{array}{l}\text { Estructura de } \\
\text { capital deseada }\end{array}$} & Capital social & $20 \%$ \\
\hline & Deuda & $30 \%$ \\
\hline & Proveedores & $15 \%$ \\
\hline & Fondos gubernamentales & $8 \%$ \\
\hline & Préstamos familiares & $7 \%$ \\
\hline & Otros o N/E & $30 \%$ \\
\hline \multirow{6}{*}{$\begin{array}{l}\text { Problemas de } \\
\text { financiamiento }\end{array}$} & Requisitos & $30 \%$ \\
\hline & Costos & $20 \%$ \\
\hline & $\begin{array}{l}\text { Falta de interés de } \\
\text { involucrados }\end{array}$ & $9 \%$ \\
\hline & Oferta insuficiente & $5 \%$ \\
\hline & Garantías y avales & $10 \%$ \\
\hline & Otros o N/E & $27 \%$ \\
\hline
\end{tabular}

Como complemento al análisis se añadieron en el cuestionario dos preguntas respecto a la percepción de la estructura de capital deseada (20\% capital propio; $30 \%$ 
deuda; $15 \%$ proveedores; $8 \%$ fondos gubernamentales; $7 \%$ préstamos familiares; $30 \%$ otros o N/E) y a las razones por las que existen problemas en las fuentes de financiamiento (30\% requisitos; $20 \%$ costos; $9 \%$ falta interés de los involucrados; $5 \%$ oferta insuficiente; $10 \%$ garantías y avales; $27 \%$ otros o N/E).

Respecto a la estructura de capital real encontrada, las proporciones medias mostradas en el cuadro 2 corroboran los resultados encontrados por el Observador Pyme referente a la baja participación del sector financiero en el otorgamiento de créditos y a las causas o problemas en el financiamiento; es decir, el desinterés hacia este sector, la falta de garantías y la falta de información en México y en otros países latinoamericanos como Argentina (Observador Pyme, 2003; EEF, 2008).

\section{Análisis}

Con la intención de validar la estructura de constructos del cuestionario, se efectuó un análisis exploratorio de los factores llevando a cabo un análisis de componentes principales con rotación Varimax para evaluar las interrelaciones entre las doce variables observadas que corresponden a los tres constructos latentes: tamaño, planeación y control. Se evaluó la adecuación muestral mediante la medida de KaiserMeyer-Olkin indicando que es adecuada si toma un valor superior a 0.5 (Bauer, 2009). Asimismo, se realizó la prueba de esfericidad de Bartlett corroborando que la matriz de correlación no es aleatoria o igual estadísticamente a la matriz identi$\operatorname{dad}\left(\mathrm{H}_{0}\right)$.

Además, se evaluó la consistencia interna de los tres constructos con múltiples indicadores (tamaño, planeación y control familiar) mediante el cálculo de sus índices o alfas de Cronbach para cada uno de ellos (Nunnally y Bernstein, 1994). El criterio por seguir (Kline, 2005) es que los valores de $\alpha$ alrededor de 0.9 son excelentes, alrededor de 0.8 son buenos, alrededor de 0.7 son adecuados, con un mínimo de 0.6 (Serva y Fuller, 2004).

Se utilizó el paquete estadístico LISREL 8.8 (Jöreskog y Sörbom, 1996) para identificar y estimar el modelo global y varios modelos considerando segmentos particulares por tipo de Pymef. Se consideraron los sectores de comercio, industria y servicios, así como las Pymef con ventas menores y mayores a un millón de dólares. Inicialmente se estimaron los modelos con las variables observadas y los constructos presentados en la figura 2. Posteriormente, y con la finalidad de mejorar el ajuste de los modelos, se procedió a calcular índices para las variables con 
indicadores múltiples (Romano et al., 2000) y se aplicó el análisis de trayectorias. Asimismo, se verificó la distribución de los índices de acuerdo con los criterios de normalidad, planteados por Hair et al. (1999), y transformaciones, sugeridos por Kline (2005).

Al evaluar el nivel de ajuste global de los modelos, se tomaron en cuenta varios índices. Se utilizó la prueba Chi cuadrada para evaluar el ajuste global, considerando que entre menor sea su valor mejor será el ajuste entre los datos y el modelo (Kline, 2005). La razón $\mathrm{NC}=$ Chi cuadrada/grados de libertad, denominada Chi cuadrada normalizada, ajusta a dicha chi cuadrada con los grados de libertad involucrados, disminuyendo el efecto del tamaño de muestra. El criterio de Bollen (1989) indica que son aceptables los valores de $\mathrm{NC}<3$. Los valores del índice de bondad de ajuste (GFI: Goodnes-of-Fit Index, Jöreskog y Sörbom, 1996) y el índice de ajuste normado (NFI: Normed Fit Index, Bentler y Bonett, 1980) se aproximan a uno cuando se tiene un buen ajuste. La cantidad de error de aproximación por grado de libertad del modelo o error de aproximación (RMSEA: Root Mean Square Error of Approximation), de acuerdo con Browne y Cudeck (1993), reflejan un buen ajuste si RMSEA $<0.05$, un ajuste razonable si $0.05<$ RMSEA $<0.08$ y un mal ajuste si RMSEA<0.10. Finalmente, se utilizó el residuo cuadrático medio estandarizado (SRMR: Standardized Root Mean Square Residual), cuyos valores ideales son los cercanos a cero (Kline, 2005).

La corroboración de las hipótesis planteadas en el modelo se llevó a cabo mediante el análisis de trayectorias (Path Análisis), la cual es una técnica particular del SEM (Jöreskog y Sörbom, 1993), en la cual se consideran solamente indicadores y relaciones causales entre éstos. Para ello, se identificaron los estimadores por utilizar en cada una de las relaciones entre variables independientes y dependientes, considerando su magnitud, signo y nivel de significancia, con el objetivo de validar las hipótesis relacionales planteadas.

\section{Resultados y discusión}

En el cuadro 3 se presentan los resultados del análisis de factores exploratorio de la muestra total, rotados, ordenados y eliminando las cargas menores a 0.4 , identificando claramente una discriminación entre los tres factores y las cargas de cada variable observada. Cabe señalar que las cargas cumplen ampliamente con el criterio mayor a 0.5; sin embargo, en el factor actitud ante el control familiar, el indicador respecto a la sucesión familiar tiene una carga ligeramente por debajo de 
ese criterio. La medida de adecuación muestral de Kaiser-Meyer-Olkin es de 0.78 y el valor Chi cuadrada para la prueba de esfericidad de Bartlett $=0.977$ con una significancia de $\mathrm{p}=0.000$, lo cual indica que el modelo factorial es apropiado. La varianza explicada por estos tres factores es de 59.9\%.

Con el objetivo de evaluar la consistencia interna de los tres constructos con múltiples indicadores (tamaño, planeación y control familiar), se calcularon las alfas de Cronbach para cada uno de ellos. En el constructo tamaño, con tres indicadores, se tuvo un valor de 0.76; en el de planeación, con cinco indicadores, 0.88 ; y en el de control familiar, 0.53 , valor que está ligeramente abajo de la referencia convencional de 0.60. Sin embargo, el análisis de fiabilidad no arrojó sugerencias que pudiesen mejorarlo mediante la eliminación de alguna de las variables observadas (Zaichkowsky, 1985), en especial el indicador respecto a la sucesión familiar, por lo que se decidió conservarlo en el análisis.

\section{Cuadro 3 \\ Cargas factoriales \\ Análisis de factores rotados Varimax}

\begin{tabular}{l|c|c|c}
\hline \multicolumn{1}{c|}{ Factores } & $\begin{array}{c}\mathbf{1} \\
\text { Planeación }\end{array}$ & $\begin{array}{c}\mathbf{2} \\
\text { Tamaño }\end{array}$ & $\begin{array}{c}\mathbf{3} \\
\text { Control }\end{array}$ \\
\cline { 2 - 4 } & 0.803 & & \\
\hline Misión y visión & 0.806 & & \\
\hline Plan estratégico & 0.824 & & \\
\hline Organigrama & 0.824 & & \\
\hline Descripción de puestos & 0.782 & & \\
\hline Capacitación formal & & & 0.737 \\
\hline Accionistas no familiares & & & 0.674 \\
\hline Administrativos no familiares & & & 0.659 \\
\hline Empleados no familiares & & & 0.432 \\
\hline Importancia de la sucesión & & 0.874 & \\
\hline Empleados & & 0.673 & \\
\hline Ventas & & 0.872 & \\
\hline Establecimientos & & &
\end{tabular}

El cuadro 4 resume los resultados de ajuste para los modelos considerados. Los indicadores del modelo global tienen, en general, un ajuste satisfactorio. La relación $\mathrm{NC}=\mathrm{Chi}^{2} / \mathrm{GL}$ es menos tres, valor recomendado por Kline (1998). Al evaluar los indicadores que definen el nivel de ajuste se observan los resultados de GFI (0.99) y NFI (0.97), los cuales tienen valores aceptables cercanos a la unidad. Finalmente, 
el RMSEA (0.066) y el SRMR (0.033) indican que las medias cuadráticas de los residuos son razonables y con buen ajuste respectivamente.

\section{Cuadro 4}

Indicadores de ajuste

de los modelos considerados

\begin{tabular}{c|c|c|c|c|c|c}
\hline Índice & $\begin{array}{c}\text { Modelo } \\
\text { global }\end{array}$ & $\begin{array}{c}\text { Modelo } \\
\text { comercio }\end{array}$ & $\begin{array}{c}\text { Modelo } \\
\text { industria }\end{array}$ & $\begin{array}{c}\text { Modelo } \\
\text { servicios }\end{array}$ & $\begin{array}{c}\text { Ventas } \\
<\mathbf{1} \mathbf{M} \\
\text { (Dlls) }\end{array}$ & $\begin{array}{c}\text { Ventas } \\
>\mathbf{1 ~ M} \\
\text { (Dlls) }\end{array}$ \\
\hline $\mathrm{N}$ & 240 & 71 & 61 & 108 & 137 & 103 \\
\hline $\mathrm{Chi}^{2}$ & 12.14 & 12.15 & 9.43 & 8.56 & 14.9 & 7.98 \\
\hline $\mathrm{Gl}$ & 6 & 6 & 9 & 6 & 6 & 6 \\
\hline $\mathrm{NC}=\mathrm{Chi}^{2} / \mathrm{gl}$ & 2.02 & 2.03 & 1.05 & 1.43 & 2.48 & 1.33 \\
\hline $\mathrm{p}-\mathrm{val}$ & 0.059 & 0.059 & 0.398 & 0.2 & 0.021 & 0.240 \\
\hline $\mathrm{GFI}$ & 0.99 & 0.96 & 0.97 & 0.98 & 0.97 & 0.98 \\
\hline $\mathrm{NFI}$ & 0.97 & 0.90 & 0.91 & 0.95 & 0.90 & 0.94 \\
\hline $\mathrm{RMSEA}$ & 0.066 & 0.130 & 0.030 & 0.065 & 0.106 & 0.058 \\
\hline $\mathrm{SRMR}$ & 0.033 & 0.067 & 0.067 & 0.050 & 0.068 & 0.050 \\
\hline
\end{tabular}

Los modelos segmentados muestran un ajuste satisfactorio considerando a $\mathrm{NC}<3$ y el GFI $>0.96$. Sin embargo, los modelos para el sector comercio y para las empresas pequeñas muestran valores relativamente bajos de NFI y altos de RMSEA simultáneamente, aunque no se llega a valores críticos.

El cuadro 5 presenta los estimadores de los coeficientes estandarizados y sus niveles de significancia de los modelos. Para el modelo global se identificó una relación positiva y significativa entre el tamaño de la empresa y la deuda como fuente de capital $\left(\mathrm{H}_{1}\right)$. Dicho hallazgo concuerda con el resultado obtenido en el estudio de Romano et al. (2000) en Australia y confirma el argumento de Berger y Udell (1998) de que, en comparación con firmas pequeñas, mientras más grande sea la empresa, ésta suscribe una cantidad mayor de deuda como fuente de financiamiento. La relación entre tamaño y deuda en las empresas se encuentra en algunos estudios sobre países desarrollados (Rajan y Zingales, 1995), en economías emergentes (Booth et al., 2001) y en Latinoamérica (Jorgensen y Terra, 2003). Dicha relación se contrapone con lo sostenido por Modigliani y Miller (1958), quienes señalan que el tamaño de la compañía no es un factor decisivo para la elección de las fuentes de capital. Se puede inferir que dicha relación, entre tamaño y deuda, es relevante para las Pymef en México. 
Se aprecia que existe una relación negativa y significativa entre la edad del director y el capital social de la empresa $\left(\mathrm{H}_{11}\right)$, la cual también respalda el resultado obtenido en Australia por Romano et al. (2000). En el caso mexicano se encontró que, del mismo modo, existe una relación positiva y significativa entre la edad del director y la utilización de las utilidades acumuladas $\left(\mathrm{H}_{12}\right)$ como fuente de financiamiento. Estos hallazgos concuerdan con las proposiciones de Van der Wijst (1989) y Matthews (1994), quienes sostienen que mientras los dueños de las pequeñas firmas envejecen, buscan financiarla por medio de fuentes internas, que no comprometan la salud financiera ni el control de la empresa. Por lo tanto, se puede inferir que en México los dueños de mayor edad de las Pymef evitan aumentar el capital social y aceptar nuevos accionistas, por lo que prefieren utilizar los recursos generados por la misma compañía para financiarse. Lo anterior soporta una de las conclusiones de la teoría sobre asimetrías de la información, ya que de acuerdo con ella los recursos internos son favorecidos sobre los externos por los dueñosdirectores de las Pymef, sobre todo en el caso de administradores que suelen ser adversos al riesgo y renuentes a perder el control de su compañía (Storey, 1994, citado por Romano et al., 2000).

Las once hipótesis restantes del modelo global no tuvieron soporte empírico significativo que permitiera justificarlas en el caso mexicano. Por lo tanto, tomando en consideración la muestra general, es decir, todos los segmentos y los tamaños de las Pymef, no existe evidencia empírica que soporte que la formalidad de la planeación administrativa y estratégica, la actitud ante el control familiar y la antigüedad de la firma tengan influencia sobre la estructura de capital de las mismas en el caso mexicano. 


\section{Cuadro 5}

Coeficientes estandarizados

y sus niveles de significancia

\begin{tabular}{|c|c|c|c|c|c|c|}
\hline Hipótesis & $\begin{array}{c}\text { Modelo } \\
\text { global }\end{array}$ & $\begin{array}{c}\text { Modelo } \\
\text { comercio }\end{array}$ & $\begin{array}{c}\text { Modelo } \\
\text { industria }\end{array}$ & $\begin{array}{c}\text { Modelo } \\
\text { servicios }\end{array}$ & $\begin{array}{c}\text { Ventas } \\
<1 \mathrm{M} \\
\text { (Dlls) }\end{array}$ & $\begin{array}{c}\text { Ventas } \\
>1 \mathrm{M} \\
\text { (Dlls) }\end{array}$ \\
\hline $\mathrm{H} 1$ & $0.13^{*}$ & -0.02 & $0.27 *$ & $0.22 * *$ & N/A & N/A \\
\hline $\mathrm{H} 2$ & -0.06 & $0.31^{* * * *}$ & -0.11 & -0.13 & N/A & N/A \\
\hline H3 & -0.03 & 0.03 & -0.09 & 0.00 & N/A & N/A \\
\hline $\mathrm{H} 4$ & 0.07 & $0.28 * *$ & -0.04 & -0.11 & $0.19^{* * *}$ & 0.00 \\
\hline $\mathrm{H} 5$ & -0.06 & -0.08 & -0.06 & 0.03 & 0.06 & -0.02 \\
\hline H6 & 0.01 & -0.10 & 0.12 & 0.05 & -0.08 & 0.12 \\
\hline $\mathrm{H} 7$ & 0.05 & -0.02 & -0.09 & 0.00 & 0.01 & -0.06 \\
\hline $\mathrm{H} 8$ & -0.02 & $0.17 *$ & 0.13 & 0.04 & -0.04 & $0.23 * * *$ \\
\hline H9 & -0.04 & 0.07 & -0.13 & -0.05 & 0.05 & -0.14 \\
\hline $\mathrm{H} 10$ & 0.02 & $0.29 * *$ & -0.22 & -0.11 & 0.09 & -0.02 \\
\hline H11 & $-0.10^{*}$ & -0.17 & -0.05 & 0.00 & -0.05 & -0.05 \\
\hline $\mathrm{H} 12$ & $0.11^{*}$ & 0.06 & $0.27 *$ & 0.08 & 0.00 & $0.26^{* *}$ \\
\hline H13 & 0.06 & -0.06 & 0.07 & 0.07 & 0.03 & 0.02 \\
\hline H14 & -0.07 & -0.06 & -0.10 & -0.08 & -0.04 & 0.01 \\
\hline$* \mathrm{p}<0.10$ & $\begin{array}{c}* * \mathrm{p}< \\
0.05\end{array}$ & $\begin{array}{c}* * * \mathrm{p}< \\
0.01\end{array}$ & & & & \\
\hline
\end{tabular}

El cuadro 5 también muestra los resultados de los modelos considerando la segmentación de la muestra por industria y por ventas. En los modelos industrial y servicios se obtuvo la misma relación positiva y significativa entre tamaño y deuda $\left(\mathrm{H}_{1}\right)$ que en el modelo global, aunque con casi el doble en una magnitud $(0.27 \mathrm{y}$ 0.22 contra 0.13 ). Por lo tanto, con base en la justificación estadística y teórica presentada anteriormente (Romano et al., 2000; Berger y Udell, 1998; Rajan y Zingales, 1995), existe evidencia que tanto en el sector industrial como en el de servicios también se respalda dicha hipótesis. De este modo, existe evidencia empírica que indica que mientras más grande sean las Pymef mexicanas tenderán a apalancarse en mayor grado. En cambio, en el sector comercio la evidencia muestra que a medida que la Pymef incrementa su tamaño, éstas tenderán a utilizar en mayor grado capital social como fuente de financiamiento $\left(\mathrm{H}_{2}\right)$. Dicha relación concuerda con Romano et al. (2000): los dueños de grandes empresas suelen considerar fuertemente el capital social como fuente de capital.

Tanto en el modelo comercial como en el de Pymef chicas (ventas $<1$ M Dlls.) existe evidencia empírica significativa que sustenta que a mayor planeación formal 
administrativa y estratégica de la empresa, mayor financiamiento a través de deuda $\left(\mathrm{H}_{4}\right)$. Esto sugiere, como sostienen varios autores, que las Pymef que no cuentan con sistemas de generación de información actualizados, ni con una planeación administrativa y estratégica eficiente que les permita respaldar y garantizar los préstamos de instituciones financieras, no obtendrán recursos para financiar sus operaciones, inversiones o llevar a cabo expansiones (Matthews et al., 1994; Chaganti et al., 1995; Berger y Udell, 1998).

Contrariamente a lo esperado y a lo encontrado por Romano et al. (2000) en Australia, se obtuvo una relación positiva y significativa entre actitud hacia el control familiar y capital social $\left(\mathrm{H}_{8}\right)$ en las Pymef del sector comercial y en las Pymef con ventas anuales mayores a un millón de dólares. Sin embargo, existen autores que proponen que los empresarios, que tienen una fuerte preferencia por mantener el control, acostumbran utilizar el capital social solamente como fuente de financiamiento siempre y cuando no represente injerencia directa en la empresa (Shrivastava y Grant, 1985; Hutchinson, 1995).

En el modelo comercial se obtuvo una relación positiva entre la edad del director y deuda $\left(\mathrm{H}_{10}\right)$. Este resultado fue inesperado, pues la dirección propuesta y justificada por la teoría indicaba una relación negativa. Factores como el tamaño muestral (71) y las particularidades del sector pudieran explicar dicho comportamiento. Sin embargo, se necesitarían estudios adicionales para poder concluir esta relación en las Pymef del sector comercial en México.

Finalmente, en los modelos industrial y ventas mayores a un millón de dólares, al igual que en el modelo global, se encontró una relación positiva entre la edad del director y las utilidades acumuladas $\left(\mathrm{H}_{12}\right)$ como fuente de financiamiento, aunque en el primero el impacto es substancialmente menor (0.11 vs 0.27 y 0.26 ). Este hallazgo coincide con las propuestas de diversos autores (Van der Wijst, 1989; Matthews, 1994; Romano et al., 2000). Por lo tanto, se puede inferir que tanto en el sector industrial como en las empresas con ventas anuales mayores a un millón de dólares los directores de mayor edad tienden a financiarse con recursos internos.

\section{Conclusiones}

Existe sustento teórico y práctico que permiten identificar ciertos factores determinantes en el tipo de financiamiento elegido por las empresas. En particular, en 
esta investigación se encontró evidencia empírica en las Pymef mexicanas, que identifican algunos de estos factores.

La presente investigación establece que las decisiones, con respecto al tipo de financiamiento elegido por este tipo de empresas, están basadas en una serie de factores internos que involucran características individuales de los dueños-directores, su experiencia, sus expectativas financieras, aversión al riesgo y actitud ante el control. Sin embargo, también existen elementos externos a la empresa y al empresario/dueño que afectan directamente su toma de decisiones de financiamiento; algunos de ellos son factores macroeconómicos, productos y mecanismos financieros, el entorno social, tecnológico y cultural, el entorno legal y fiscal, y el entorno internacional.

Se sugiere ampliar el alcance de la investigación mediante estudios que incorporen un mayor número de características de la empresa familiar, detallando más su plan de sucesión, plan de compensación a familiares y separación negocio-familia, ética, entre otros (Domenge, 2008).

Los estudios de los factores que afectan las decisiones de financiamiento en la Pymef mexicana pueden sentar las bases para que estas empresas, las instituciones financieras y los organismos gubernamentales puedan diseñar e implementar conjuntamente programas y productos financieros que permitan satisfacer adecuadamente las demandas de financiamiento de este sector.

\section{Referencias}

Barton, S.L. y P.J.Gordon (1987). Corporate strategy: Useful perspective for the study of capital structure? Academy of Management Review 12 (1): 67-75.

Bauer Collage of Business. University of Houston. Factor analysis. Disponible en http://www.bauer.uh.edu/nbsyam/documents/MktRes-MARK4338-Lecture10-11_003.ppt\#262,6,Chapter Outline.

Bentler, P.M. y D.G. Bonett (1980). Significance test and goodness of fit in the analysis of covariance structures. Psychological Bulletin 88 (3): 591-606. 
Belausteguigoitia, R.I. (2003). Empresas familiares: su dinámica, equilibrio y consolidación. México: McGraw-Hill Interamericana.

Berger, A.N. y G.F. Udell (1998). The economics of small business finance: The roles of private equity and debt markets in the financial growth cycle. Journal of Banking y Finance (22): 613-673.

Bertrand, M. y A. Schoar (2002). Managing with style: the effect of managers on firm policies. Working paper, IL, USA: University of Chicago.

Bollen, K.A. (1989). Structural equations with latent variables. USA: Wiley.

Booth, L., A. Aivazian, A. Demirguc-Kunt y V. Maksimovic (2001). Capital structure in developing countries. Journal of Finance 56 (1): 87-130.

Brockhaus, R.H. (1994). Entrepreneurship and family business research: Comparisons, critique, and lessons. Entrepreneurship Theory y Practice 19 (1): 25-38.

Browne, M.W. y R. Cudeck (1993). Alternative ways of assessing model fit. En K.A. Bollen y J.S. Long. Testing structural equation models. USA: SAGE.

Cassar, G. y S. Holmes (2003). Capital structure and financing of SMEs: Australian evidence. Accounting and Finance (43): 123-147.

Chaganti, R., D. DeCarolis y D. Deeds (1995). Predictors of capital structure in small ventures. Entrepreneurship Theory and Practice (20): 7-18.

Chittenden, F., G. Hall y P. Hutchinson (1996). Small firm growth, access to capital markets and financial structure: Review of issues and an empirical investigation. Small Business Economics (8): 59-67.

Delgado, L.P., C. Ramírez e I. Anaya (2006). Factores financieros de éxito y fracaso involucrados en la creación de empresas. Documento interno. México: ITAM.

Dollinger, M.J. (1995). Entrepreneurship: strategies and resources. USA: Irwin. 
Domenge, R. (2008). PyMEs: Éxito y fracaso en México. Contaduría Pública 37 (434): 12-16.

EEF, Encuesta de Empresas Familiares en México. 2008. México: Banamex-Profit Consulting.

García B. J. y P. García (2007). Factores determinantes de la estructura de capital en la Pequeña y Mediana Empresa familiar mexicana. Documento interno. México: ITAM.

Hair, J.F., R.E. Anderson, R.L. Tatham y W.C. Black (1999). Análisis multivariante. Madrid: Prentice Hall Iberia: 611-705.

Harvey, M. y R. Evans (1995). Forgotten sources of capital for the family-owned business. Family Business Review 8 (3): 159-175.

Hutchinson, R.W. (1995). The capital structure and investment decision of the small owner-managed firm: Some exploratory issues. Small Business Economics (7): 231-239.

Instituto Nacional de Estadística, Geografía e Informática (2006). Micro, Pequeña, Mediana y Gran Empresa Estratificación de los Establecimientos Censos Económicos 2004. México: INEGI.

Jones, R.G. (1979). Analyzing initial and growth financing for small business. Management Accounting (61): 30-38

Jorgensen, J.J. y P.R. Terra (2003). Determinants of capital structure in Latin America: The role of firm-specific and macrioeconomics factors. Working Paper.

Jöreskog, K.G. y D. Sörbom (1996). LISREL 8: Structural equation modeling. Chicago: Scientific Software International.

Kline, Rex B. (2005). Principles and practice of structural equation modeling. Second Edition. USA: The Guilford Press.

Kuhlman, R. (1997). Negocios vs. Familia. Entrepreneur 5 (12): 28-29. 
Levin, R.I. y V.P. Travis (1987). Small company financing: What the books don't say. Harvard Business Review noviembre-diciembre: 30-32.

Matthews, C.H., D.P. Vasudevan, S.L. Barton y R. Apana (1994). Capital structure decision making in privately held firms: Beyond the finance paradigm. Family Business Review 7 (4): 349-367.

Modigliani, F. y M.H. Miller (1958). The cost of capital, corporate finance and the theory of investment. American Economic Review junio: 261-297.

Myers, S.C. (1984). The capital structure puzzle. Journal of Finance 39 (3): $575-592$.

Nunnally, J.C. e I.H. Bernstein (1994). Psychometric theory. Third Edition. USA: McGraw Hill.

Observatorio Pyme. 2003. Disponible en http://www.cipi.gob.mx/html/principalesresultados.pdf

Organización para la Cooperación y Desarrollo Económico, (2007). SME's in Mexico: Issues and Policies. Paris: OECD Publishing.

Poutziouris, P., F.Chittenden y N. Michaelas (1998). The financial affairs of private companies. Manchester Business School, The University of Manchester.

Prasad, S., Ch. Green y V. Murinde (2001). Company financing, capital structure and ownership: a survey, and implications for developing economies. Centre for International, Financial and Economics Research. Department of Economics, UK: Loughborough University.

Rajan, R.G. y L. Zingales (1995). What do we know about capital structure? Some evidence from internacional data. The Journal of Finance 50 (5): 1421-1460.

Romano, C., G. Tanewski y P. Smyrnios (2000). Capital structure decision making: a model for family business. Journal of Business Venturing (16): 285-310. 
Sander, P. (1998). Capital structure choice in estonian companies: a survey. Estonia: University of Tartu.

Serva, M.A. y M.A. Fuller (2004). Aligning what we do and what we measure in business schools: incorporating active learning and effective media use in the assessment of the instruction. Journal of Management education 28 (1): 19-38.

Shrivastava, P. y J.H. Grant (1985). Empirically derived models of strategic decision making processes. Strategy Management Journal 6 (2): 97-113.

Smith, C.W. (1986). Investment banking and the capital acquisition process. Journal of Financial Economics january: 3-25.

Smyrnios, K.X., G.A. Tanewski y C. Romano (1998). Development of a measure of the characteristics of family business. Family Business Review 11 (1): 49-60.

Sonnenfeld, J.A. y P.L. Spence (1989). The parting patriarch of a family firm. Family Business Review 2 (4): 355-375.

Storey, D.J. (1994). Understanding the small business sector. Londres: Routledge.

Tenanhaus, M. (2007). Structural equation modelling for small samples. France: HEC School of Management.

Titman, S. y S. Tsyplakov (2005). A dynamic model of optimal capital structure. Working paper.

Titman, Sh. y R. Wessels (1988). The determinants of capital structure choice. Journal of Finance 43 (1): 1-19.

Thompson, A. y A.J. Strickland (1999) Administración estratégica: conceptos y casos. México: McGraw Hill.

Van der Wijst, D. (1989). Financial structure in small business: Theory, tests and applications. Berlín: Springer-Verlag. 
Zaichkowsky, J.L. (1985). Measuring the involvement concept. Journal of Consumer Research 12 (3): 341-352.

Zimmerer, T. y N. Scarborough (2002). Essentials of entrepreneurship and small business management. New Jersey: Prentice Hall. 


\section{Anexo 1}

\section{Cuestionario}

1. ¿Es familiar la empresa donde trabaja o de la que es dueño?: Sí No ( $\mathrm{Si}$ contesta No se termina el cuestionario)

2. ¿Cuál es su puesto dentro de la empresa? (Si contesta 1 ó 2 se termina el cuestionario)

[5] Dueño [4] Director [3] Gerente [2] Empleado [1] Obrero

3. ¿Con cuántos años de experiencia profesional cuenta el director/gerente general?

4. ¿Dentro de qué sector empresarial catalogaría su empresa?

[1] Industrial/Manufacturero [4] Comercial/Mayorista/Minorista

[2] Financiero/Asesoría/Consultoría [5] Hotelero/Restaurantero/Turístico

[3] Educativo/Capacitación [6] Otro

5. ¿Cuántos años de fundada tiene la empresa?

6. ¿Cuántos empleados tiene la empresa?

7. ¿Con cuántos establecimientos cuenta la empresa?

8. ¿Cuáles fueron sus ventas anuales (en pesos) aproximadas durante 2006?

[1] Hasta 5 millones [2] Entre 5 y 10 millones [3] Entre 10 y 15 millones [4] Entre 15 y 20 millones [5] Entre 20 y 25 millones [6] Más de 25 millones 
9. De acuerdo con la estructura de capital de la empresa, asigne la proporción a cada uno de los siguientes rubros:

\begin{tabular}{|c|c|}
\hline (1) Capital (capital social) & \\
\hline (2) Deuda (préstamos bancarios, proveedores, otros) & \\
\hline (3) Utilidades retenidas (utilidad acumulada) & \\
\hline (4) Préstamos familiares & \\
\hline Total & $100 \%$ \\
\hline
\end{tabular}

10. Marque en qué grado está usted de acuerdo con los siguientes enunciados (1 es el mínimo, 5 es el máximo):

a) La misión y visión de la empresa ha sido desarrollada por escrito.

b) Un plano estratégico de largo plazo ha sido desarrollado por escrito.

c) El organigrama ha sido documentado por escrito.

d) Existen descripciones de los puestos gerenciales por escrito.

e) Miembros del equipo administrativo participan en cursos y entrenamientos.

f) Los dueños aceptarían accionistas NO familiares.

g) Los dueños aceptarían administradores NO familiares en los puestos clave.

h) Un objetivo principal de la empresa es emplear a miembros de la familia.

i) Un objetivo principal de la empresa es continuar funcionando con la siguiente generación.

11. ¿Cuál es la edad aproximada del director/gerente general de la empresa? [1] 20-30 [2] 31-40 [3] 41-50 [4] 51-60 [5] Más de 60

12. ¿Cuál es el grado máximo de estudios del director/gerente general de la empresa?

[1] Secundaria [2] Preparatoria [3] Carrera técnica [4] Licenciatura [5] Posgrado

13. ¿Cuál cree que sea el mejor tipo de financiamiento para su empresa y por qué?

14. ¿Cuál cree que sea el mayor problema para el financiamiento de una Pyme?

Nombre de la compañía (opcional): 